\title{
Applied pressure of cooling gas: a neglected determinant for size of specimens in transbronchial cryobiopsy
}

\author{
Xiaobo Chen", Shiyue Li", Weiquan Xiao, Yao Yao, Yanqiuzi Cheng, Yongshun Ye, Huanjie Chen, \\ Zhuquan Su, Nanshan Zhong
}

State Key Laboratory of Respiratory Disease, National Clinical Research Center for Respiratory Disease, Guangzhou Institute of Respiratory Health, The First Affiliated Hospital of Guangzhou Medical University, Guangzhou, China

Contributions: (I) Conception and design: N Zhong; (II) Administrative support: None; (III) Provision of study materials or patients: None; (IV) Collection and assembly of data: X Chen, S Li, W Xiao; (V) Data analysis and interpretation: X Chen, S Li, W Xiao; (VI) Manuscript writing: All authors; (VII) Final approval of manuscript: All authors.

\#These authors contributed equally to this work.

Correspondence to: Nanshan Zhong. State Key Laboratory of Respiratory Disease, National Clinical Research Center for Respiratory Disease, Guangzhou Institute of Respiratory Health, The First Affiliated Hospital of Guangzhou Medical University, 151 Yan jiang Rd., Guangzhou 510120, China. Email: nanshan@vip.163.com.

Background: Transbronchial cryobiopsy (TBCB) is an option to surgical biopsy for the diagnosis in interstitial lung diseases. Several impact factors have received wide attention, including the freezing time, cryoprobe size, and contact pressure. However, the effect of the applied gas pressure on the specimen size has not been well elucidated. The purpose of this study is to investigate the effect of the applied gas pressure on the TBCB specimen size.

Methods: Cryoprobes with a diameter of $1.9 \mathrm{~mm}$ were used to perform TBCB on 4 beagle canines under general anesthesia. TBCB was performed with a total of 16 time-pressure combinations that were randomly combined with 4 freezing times $(3,4,5$, and 6 s) and 4 gas pressures (40, 50, 55, and 60 bar). For each combination, 8 biopsies were performed. The size and quality of specimens, as well as complications, were evaluated.

Results: A total of 128 TBCB specimens were obtained. With the same freezing time, the specimen sizes obtained by different applied gas pressures were significantly different $(\mathrm{P}<0.05)$ and positively correlated with the gas pressures (r: 0.797-0.867). With the same gas pressure, the size of the TBCB specimens was positively correlated with the freezing time ( $\mathrm{r}$ : 0.503-0.752). In the 40-bar group, no tissues were obtained when the freezing times were 3-5 s. In the 50-bar and 55-bar groups, qualified specimens were obtained when the freezing times were 5 and $6 \mathrm{~s}$. In the 60-bar group, qualified specimens were obtained when the freezing times were 3-6 s.

Conclusions: The TBCB specimen size was positively correlated with the applied gas pressure. The applied gas pressure contributed to the sample size and quality. To obtain qualified specimens with a $1.9-\mathrm{mm}$ cryoprobe during TBCB, the lowest limit of the normal working gas pressure range should be increased to greater than 50 bar.

Keywords: Transbronchial cryobiopsy (TBCB); specimen size; gas pressure; animal

Submitted Jul 15, 2020. Accepted for publication Feb 10, 2021.

doi: $10.21037 /$ jtd-20-2446

View this article at: http://dx.doi.org/10.21037/jtd-20-2446 


\section{Introduction}

Transbronchial cryobiopsy (TBCB) represents an essential diagnostic step in interstitial lung disease (ILD) to obtain sufficiently larger tissue samples, compared to conventional forceps (1-3). It is known that tissue samples have a positive correlation with freezing time, and patients have a higher risk of TBCB complications when obtaining larger tissue samples (4). However, to obtain a larger tissue sample to improve the diagnostic yield in ILD is contradicted for the safety of TBCB in clinical work. Unfortunately, the balance between the acceptable sample size and the minimal risk of complications remains unknown. In this study, an animal study is conducted to explore the effect of different gas pressures on the TBCB specimen size under the same conditions (time, probe, and contact pressure).

We present the following article/case in accordance with the ARRIVE Guidelines Checklist (available at http:// dx.doi.org/10.21037/jtd-20-2446).

\section{Materials}

\section{The experimental design}

All of the canines ( $\mathrm{n}=4,10-12 \mathrm{~kg}, 270-320$ days) were purchased from Nanjing Chaimen Biological Technology Co., Ltd. (Nanjing, China). The procedures were conducted at the Animal Experimental Center of Guangzhou Medical University (Guangzhou, China). According to the committee's recommendation for reducing the number of animals, all of the canines were performed TBCB on them with the working pressure at various freezing times, and 8 biopsies were obtained under general anesthesia. Consistent with the clinical standard of the working pressure (40-60 bar) of the gas source, each canine was performed TBCB in the order from a lower to higher working pressure (40, 50, 55, and 60 bar). For each working pressure, 8 biopsies were performed at each freezing time (3, 4,5 , and 6 s). The size and quality of the specimens, as well as complications, were evaluated. Animal experiments were performed under a project license (\# SYSK201701710002) granted by institutional ethics board of Animal Protection Ethics Committee of Longguixingke Animal Farm, Baiyun District, Guangzhou, in compliance with the guideline of Animal Ethics Committee Longguixingke Animal Farm for the care and use of animals.

\section{Preparation of the beagle canines}

Beagle canines were fasted for 8 hours prior to surgery.
Anesthesia was induced via intramuscular injection of ketamine $(1 \mathrm{mg} / \mathrm{kg})$ combined with femoral intravenous injection of $3 \%$ pentobarbital sodium $(0.5 \mathrm{~mL} / \mathrm{kg})$. After anesthesia, the canines were placed on the experimental table in the supine position. Self-breathing was maintained, and cardiopulmonary monitoring was conducted. A tracheal tube with a balloon (ID 8.0) was selected for endotracheal intubation guided by bronchoscopy, and the insertion depth of the catheter was adjusted to $3-4 \mathrm{~cm}$ above the carina.

\section{Experimental TBCB procedure}

TBCB was performed by physicians experienced in bronchoscopy, who had performed more than $50 \mathrm{TBCB}$ procedures in clinical practice. Prior to the procedures, the cryosurgical system (Erbokryo CA, ERBE, Germany) with the recommended range of working gas pressure (40-60 bar) and the flexible cryoprobes (diameter $1.9 \mathrm{~mm}$, ERBE, Germany) were tested in vitro to ensure normal conditions. The canines underwent routine airway examinations with a flexible bronchoscope with an outer diameter of $4.9 \mathrm{~mm}$ (Olympus, Japan). The flexible cryoprobes were passed through the bronchoscope working channel to the target bronchus for the biopsy and positioned under $\mathrm{X}$-ray guidance. Once resistance was encountered, the cryoprobe was pulled back $1 \mathrm{~cm}$, and the TBCB was performed for various freezing times as described above. After cooling, the bronchoscope and the cryoprobe were pulled back simultaneously. The biopsy specimens were collected and measured by the assistant. Prior to another TBCB procedure, bleeding was recorded for a maximum of 10 minutes. The number of tissues obtained by the biopsy was under various conditions as described above. The assistant recorded the size of each biopsy specimen and complications such as bleeding and pneumothorax. Based on the previous studies described (4), the bleeding was recorded as follows: grade 0 (no bleeding), grade 1 (negative pressure suction could stop bleeding without additional hemostatic measures), grade 2 (local injection of frozen saline/epinephrine or balloon occlusion was required to stop bleeding), and grade 3 (severe bleeding that could not be controlled under endoscopy, resulting in animal death).

\section{Specimen measurement and processing}

Specimens were acquired and immediately fixed in $10 \%$ neutral buffered formalin for 24 hours and then were dehydrated, cleared in xylene, and embedded in paraffin. 
Table 1 The specimen sizes and gas pressures according to application time

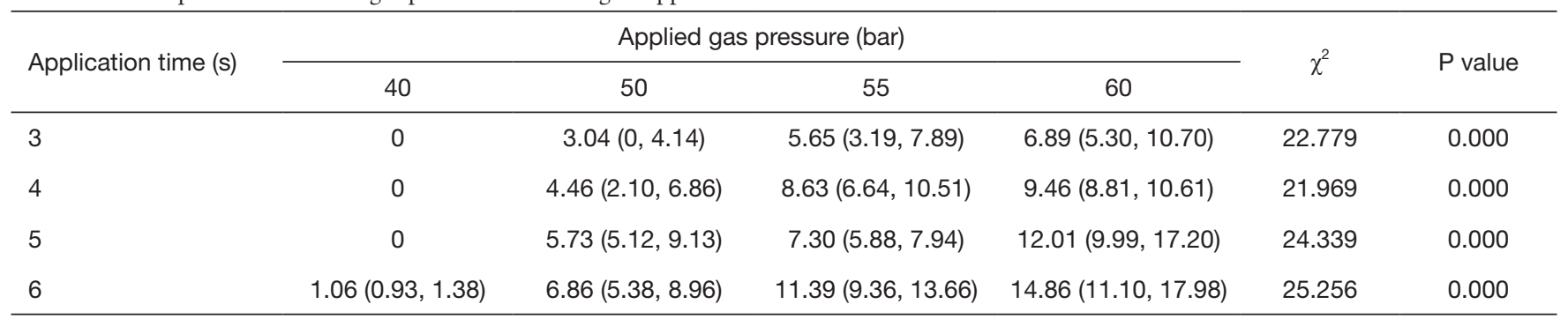

The specimen sizes are presented as square millimeters $\left(\mathrm{mm}^{2}\right)$.

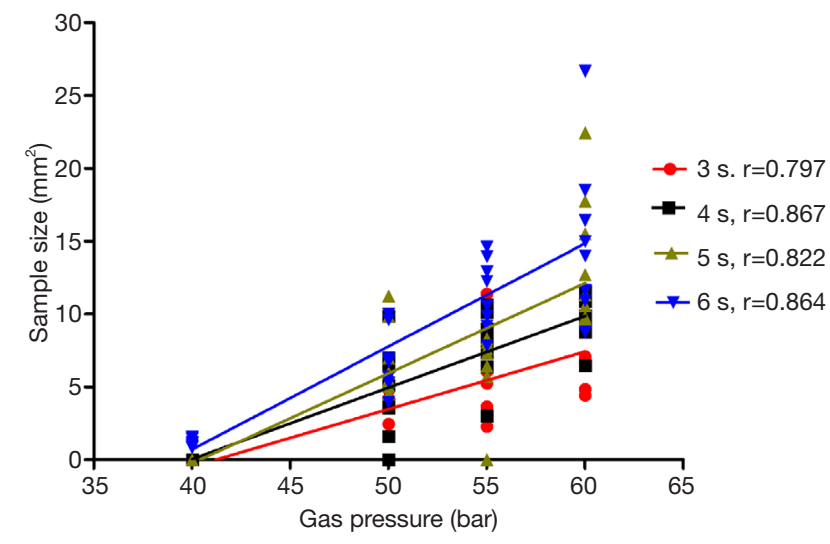

Figure 1 Correlation between the specimen sizes and applied gas pressures according to freezing time.

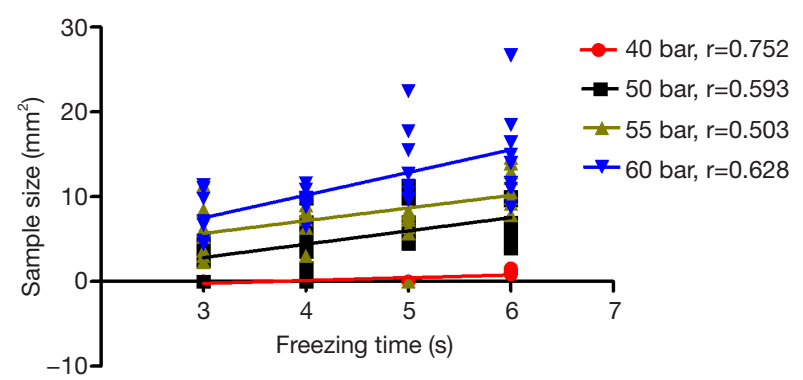

Figure 2 Correlation between the specimen sizes and freezing times according to applied gas pressure.

The paraffin blocks were sectioned at $3 \mu \mathrm{m}$ intervals using a paraffin microtome. Hematoxylin and eosin (HE) staining was performed on these sections before they were mounted in neutral balsam. The pathological sections were scanned using a Digital Pathology Scanner (PRECICE 500B, UNIC Technologies Inc., China). An iViewer digital slide browser (iViewer 6.2.3, UNIC Technologies Inc., China) was used to measure the maximum cross-sectional area, which was recorded as the specimen area. The evaluating system for specimen quality was based on that described in the literature (4) with revisions as follows: the specimen quality was evaluated by the integrity of the specimen, whether the tissue was crushed, and whether the alveoli, terminal bronchioles, and small blood vessels were included. If the tissue was not crushed, and the alveoli, terminal bronchioles, and small blood vessels were all included, the specimens were considered qualified. The proportion of qualified specimens to the total number of specimens is qualification.

\section{Statistics}

The statistical analyses were performed using SPSS 16.0 statistical software. The measurement data are described as the median (interquartile range). A comparison of the specimen sizes obtained under the different gas pressures was performed using the multi-sample nonparametric test. The correlations of specimen size with gas pressure and freezing time were analyzed by a linear regression. Differences with a value of $\mathrm{P}<0.05$ were considered to be statistically significant.

\section{Results}

\section{Relationships of specimen size with applied gas pressure and freezing time}

Using the same freezing times, the differences in the TBCB specimen sizes due to the use of different gas pressures were significant (Table $1, \mathrm{P}<0.01$ ) and were positively correlated with the gas pressure (r: 0.797-0.867, Figure 1). When using the same applied gas pressure, the TBCB specimen size was positively correlated with the freezing time (r: $0.503-0.752$, Figure 2). 
Table 2 Histological quality of cryobiopsy sample with the different gas pressure at different freezing time

\begin{tabular}{|c|c|c|c|c|c|c|c|c|}
\hline Histology & \multicolumn{4}{|c|}{50 bar $(n=8), n(\%)$} & \multicolumn{4}{|c|}{55 bar $(n=8), n(\%)$} \\
\hline Small arterioles & $6(75.0)$ & 7 (87.5) & $8(100.0)$ & $8(100.0)$ & $7(87.5)$ & $8(100.0)$ & $8(100.0)$ & $8(100.0)$ \\
\hline Terminal bronchioles & $3(37.5)$ & $5(62.5)$ & $8(100.0)$ & $8(100.0)$ & $6(75.0)$ & $7(87.5)$ & $8(100.0)$ & $8(100.0)$ \\
\hline Alveolar tissue & $7(87.5)$ & $8(100.0)$ & $8(100.0)$ & $8(100.0)$ & $8(100.0)$ & $8(100.0)$ & $8(100.0)$ & $8(100.0)$ \\
\hline Qualification rate* & $2(25.0)$ & $5(62.5)$ & $8(100.0)$ & $8(100.0)$ & $6(75.0)$ & 7 (87.5) & $8(100.0)$ & $8(100.0)$ \\
\hline
\end{tabular}

${ }^{*}$, if the tissue was not crushed, and the alveoli, terminal bronchioles, and small blood vessels were all included, the specimens were considered qualified.

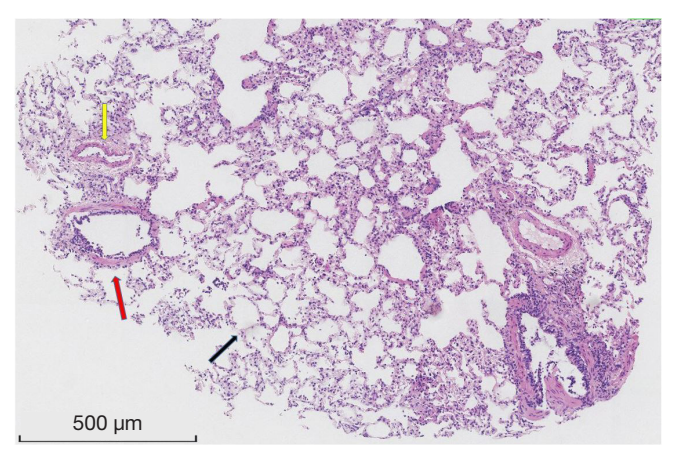

Figure 3 Histological quality of cryobiopsy sample at a gas pressure of 60 bar and freezing time of $4 \mathrm{~s}$. Pathological section stained in haematoxylin and eosin. Normal alveoli (black arrow), terminal bronchiole (red arrow) and arteriole (yellow arrow) were observed.

Table 3 Complications of different gas pressures

\begin{tabular}{lcccc}
\hline \multirow{2}{*}{$\begin{array}{l}\text { Complication } \\
\text { (bleeding) }\end{array}$} & 40 & 50 & 55 & 60 \\
\hline \cline { 2 - 5 } Grade 0 & 29 & 23 & 21 & 17 \\
Grade 1 & 3 & 7 & 8 & 12 \\
Grade 2 & 0 & 2 & 3 & 3 \\
Grade 3 & 0 & 0 & 0 & 0 \\
Pneumothorax & 0 & 0 & 1 & 2 \\
\hline
\end{tabular}

\section{Condition and quality of the acquired specimens}

A total of 128 TBCBs were performed. At a gas pressure of $40 \mathrm{bar}$, no tissue was obtained when the freezing times were $3-5 \mathrm{~s}$, and only a small amount of bronchial mucosa was obtained when the freezing time was $6 \mathrm{~s}$. At gas pressures of
50 and 55 bar, qualified specimens were obtained when the freezing times were 5 and $6 \mathrm{~s}$ (Table 2). At a gas pressure of $60 \mathrm{bar}$, qualified specimens were obtained when the freezing times were 3-6 s (Figure 3), and the qualification rate of the sample was $100 \%$.

\section{Complications}

In this study, mild to moderate bleeding (grades 1 and 2) was observed in 38 TBCBs (29.7\% of $128 \mathrm{TBCBs})$, and no severe fatal bleeding (grade 3 ) occurred. Among the biopsies, grade 1 bleeding occurred 30 times, and grade 2 bleeding occurred 8 times. No significant correlation was found between the number of occurrences, severity of bleeding, and applied gas pressure. With a gas pressure of $60 \mathrm{bar}$, pneumothorax occurred once with a freezing time of $4 \mathrm{~s}$ and once with a freezing time of $6 \mathrm{~s}$. With a gas pressure of 55 bar, pneumothorax occurred only once with a freezing time of $5 \mathrm{~s}$, and after decompression, the TBCB was successfully continued (Table 3).

\section{Discussion}

Growing evidence suggests that the utility of ТВСВ has shown that it can more effectively identify complex histological patterns for ILD diagnosis. Compared to conventional forceps hampered by the small tissue size, TBCB has the advantage of obtaining a larger and complete tissue structure specimen, which also contributes to a high diagnostic efficiency $(3,5)$. However, a larger tissue for improving the ILD diagnostic yield usually means a higher risk of complications during the TBCB procedure. A previous study (6) reported that the impact factors for TBCB specimen size were freezing time, cryoprobe 
diameter, and probe-tissue contact pressure, as well as tissue properties. In this work, a neglected impact factor on the specimen size during the TBCB procedure in clinical work, and this showed that the TBCB specimen size was positively correlated with the applied gas pressure ( $\mathrm{r}: 0.797-0.867$ ).

This study showed that gas pressures played a role in the TBCB specimen size in canines. The results showed that all other conditions being equal, the specimen size was positively correlated with the applied gas pressure, which is a neglected impact factor in application. Additionally, these results suggested that the specimen size was positively correlated with the freezing time with all other conditions being equal. These results agreed with previous studies $(4,6)$.

Cryobiopsy was used for the biopsy of the endobronchial lesions for the first time in 2008 (7). Then, TBCB initially was used to diagnose pulmonary lesions of ILD in 2009 (1). Recently, this technique has been extensively used to diagnose ILD (8), peripheral pulmonary nodules (9), and rejection after lung transplantation (10) due to its excellent safety and effectiveness, particularly for the pathological classification diagnosis of ILD. Studies $(4,6)$ have shown that the size of the TBCB is correlated with the freezing time, cryoprobe, tissue consistency, and contact pressure of the probe. However, until now there existed no published research regarding the cooling gas pressure.

Generally, studies (11-13) have on TBCB procedures with freezing times of 3-6 s, but no further exploration has been for gas pressures, and it has been recommended to use a relatively broad range of 40-60 bar. Importantly, the applied gas pressure has been neglected in previous studies $(1,14-16)$, and flexible cryoprobes with the same diameter, type of cooling gas, and freezing time were used, and the specimen sizes were still varied $\left(9-15.11 \mathrm{~mm}^{2}\right)$. The results of this study showed that when the applied gas pressure was less than 50 bar, qualified specimens could not be obtained under the commonly used freezing times with a $1.9-\mathrm{mm}$ cryoprobe. In contrast, with a pressure greater than 50 bar, the increase in cooling pressure was positively correlated with adequate and larger specimens.

Notably, a previous study (4) suggested that for TBCB, $3 \mathrm{~s}$ might be an acceptable freezing time to obtain qualified specimens with a minimal complication rate. It is inaccurate to state that the complication rate during the TBCB procedure only depends on freezing time, and various factors, including gas pressure, contribute to the complications. In clinical TBCB procedures, gas pressure has typically been a neglected factor with regard to the complications.
A question remains: why is the specimen size positively correlated with the gas pressure? Freezing equipment is applied in medicine based on the Joule-Thompson effect, with $\mathrm{CO}_{2}$ as the gas source. As high-pressure $\mathrm{CO}_{2}$ gas is released through small holes, and due to throttle expansion (the Joule-Thompson effect), the temperature of the cryoprobe rapidly cools. Therefore, the higher the gas pressure, the better efficacy of the cooling, and the larger size of the specimen. It is worthwhile to mention that previous studies have not noted this type of impact. Before TBCB was used in clinical practice, the primary purpose of the original design of the freezing equipment was only the freeze-thaw action. Because the freezing time of the freezethaw is much longer than the TBCB procedure, a reduction in performance can be compensated by an extension of time. The temperature for the freeze-thaw cycle is within a certain range time, and the requirement for the temperature decrease rate per unit time is not particularly high; therefore, it is difficult to observe the small changes in local temperature on the surface. Thus, in clinical application, the effect of a decrease in gas pressure within the recommended range on the freeze-thaw action is minor or difficult to notice.

The limitations of the study should be taken into consideration. This study used animal experiments, and some differences in lung tissues exist between beagle canines and humans, especially for lung tissues with different lesions. In addition, the evaluation method for the quality of specimens may not be directly applicable to lung tissues with different diseases. The small numbers of TBCB samples limited the statistical power, especially the safety of procedure. Manufacturer suggested that a 1.9-mm cryoprobe could be reused for 30 times within recommended working pressure. But still there was a very low rate of a buckling in the probe for each procedure. The effect of the applied gas pressure on clinical TBCB still requires further confirmation by a multicenter prospective randomized controlled study.

In summary, the TBCB specimen size was positively correlated with the applied gas pressure; therefore, the applied gas pressure is an important factor that influences the sample size and quality and should not be neglected. When using a $1.9-\mathrm{mm}$ cryoprobe for TBCB, the lowest limit of the normal working pressure range of gas should be increased to above 50 bar to obtain qualified specimens. The optimal combination of applied gas pressure and freezing time in different disease types will be further explored. 


\section{Acknowledgments}

We acknowledge Guangqiao Zeng and Qian Han for their assistance with proofreading the manuscript.

Funding: Medical research Foundation of Guangdong (sponsor: Xiaobo Chen, A2020106).

\section{Footnote}

Reporting Checklist: The authors have completed the ARRIVE Guidelines Checklist. Available at http://dx.doi. org/10.21037/jtd-20-2446

Data Sharing Statement: Available at http://dx.doi. org/10.21037/jtd-20-2446

Conflicts of Interest: All authors have completed the ICMJE uniform disclosure form (available at http://dx.doi. org/10.21037/jtd-20-2446). Prof. NZ serves as the editorin-chief of Fournal of Thoracic Disease. The authors have no other conflicts of interest to declare.

Ethical Statement: The authors are accountable for all aspects of the work in ensuring that questions related to the accuracy or integrity of any part of the work are appropriately investigated and resolved. Animal experiments were performed under a project license (\# SYSK201701710002) granted by institutional ethics board of Animal Protection Ethics Committee of Longguixingke Animal Farm, Baiyun District, Guangzhou, in compliance with the guideline of Animal Ethics Committee Longguixingke Animal Farm for the care and use of animals.

Open Access Statement: This is an Open Access article distributed in accordance with the Creative Commons Attribution-NonCommercial-NoDerivs 4.0 International License (CC BY-NC-ND 4.0), which permits the noncommercial replication and distribution of the article with the strict proviso that no changes or edits are made and the original work is properly cited (including links to both the formal publication through the relevant DOI and the license). See: https://creativecommons.org/licenses/by-nc-nd/4.0/.

\section{References}

1. Babiak A, Hetzel J, Krishna G, et al. Transbronchial cryobiopsy: a new tool for lung biopsies. Respiration
2009;78:203-8.

2. Griff S, Ammenwerth W, Schönfeld N, et al.

Morphometrical analysis of transbronchial cryobiopsies.

Diagn Pathol 2011;6:53. Erratum in: Diagn Pathol 2016 19;11:64. doi: 10.1186/s13000-016-0515-1.

3. Pajares V, Puzo C, Castillo D, et al. Diagnostic yield of transbronchial cryobiopsy in interstitial lung disease: a randomized trial. Respirology 2014;19:900-6.

4. Ing M, Oliver RA, Oliver BG, et al. Evaluation of Transbronchial Lung Cryobiopsy Size and Freezing Time: A Prognostic Animal Study. Respiration 2016;92:34-9.

5. Ganganah O, Guo SL, Chiniah M, et al. Efficacy and safety of cryobiopsy versus forceps biopsy for interstitial lung diseases and lung tumours: A systematic review and meta-analysis. Respirology 2016;21:834-41.

6. Franke KJ, Szyrach M, Nilius G, et al. Experimental study on biopsy sampling using new flexible cryoprobes: influence of activation time, probe size, tissue consistency, and contact pressure of the probe on the size of the biopsy specimen. Lung 2009;187:253-9.

7. Hetzel J, Hetzel M, Hasel C, et al. Old meets modern: the use of traditional cryoprobes in the age of molecular biology. Respiration 2008;76:193-7.

8. Johannson KA, Marcoux VS, Ronksley PE, et al. Diagnostic Yield and Complications of Transbronchial Lung Cryobiopsy for Interstitial Lung Disease. A Systematic Review and Metaanalysis. Ann Am Thorac Soc 2016;13:1828-38.

9. Schuhmann M, Bostanci K, Bugalho A, et al. Endobronchial ultrasound-guided cryobiopsies in peripheral pulmonary lesions: a feasibility study. Eur Respir J 2014;43:233-9.

10. Yarmus L, Akulian J, Gilbert C, et al. Cryoprobe transbronchial lung biopsy in patients after lung transplantation: a pilot safety study. Chest 2013;143:621-6.

11. Kropski JA, Pritchett JM, Mason WR, et al. Bronchoscopic cryobiopsy for the diagnosis of diffuse parenchymal lung disease. PLoS One 2013;8:e78674.

12. Griff S, Schönfeld N, Ammenwerth W, et al. Diagnostic yield of transbronchial cryobiopsy in non-neoplastic lung disease: a retrospective case series. BMC Pulm Med 2014;14:171.

13. Hernández-González F, Lucena CM, Ramírez J, et al. Cryobiopsy in the diagnosis of diffuse interstitial lung disease: yield and cost-effectiveness analysis. Arch Bronconeumol 2015;51:261-7.

14. Fruchter O, Fridel L, El Raouf BA, et al. Histological diagnosis of interstitial lung diseases by cryo- 
transbronchial biopsy. Respirology 2014;19:683-8.

15. Fruchter O, Fridel L, Rosengarten D, et al. Transbronchial cryobiopsy in immunocompromised patients with pulmonary infiltrates: a pilot study. Lung 2013;191:619-24.
16. Fruchter O, Fridel L, Rosengarten D, et al. Transbronchial cryo-biopsy in lung transplantation patients: first report. Respirology 2013;18:669-73.
Cite this article as: Chen X, Li S, Xiao W, Yao Y, Cheng Y, Ye Y, Chen H, Su Z, Zhong N. Applied pressure of cooling gas: a neglected determinant for size of specimens in transbronchial cryobiopsy. J Thorac Dis 2021;13(4):2099-2105. doi: 10.21037/jtd20-2446 